\title{
Acreditación Institucional y la mirada de los actores. Un estudio cualitativo en universidades privadas de Santiago*
}

\author{
Institutional accreditation and the actors' perception. A qualitative study \\ in private universities of Santiago
}
Acreditação Institucional e o olhar dos atores. Um estudo qualitativo em universida- des privadas em Santiago

\author{
Emilio Torres Rojas \\ Escuela de Sociología, Universidad Central. Telf.: 5826513-76672611. \\ Correo electrónico: etorres@ucentral.cl
}

\begin{abstract}
RESUMEN
El aseguramiento de la calidad mediante sistemas de acreditación es un desafío que confronta a universidades y actores. La evidencia empírica en Latinoamérica y en Chile es aún reducida, desconociéndose cómo se construye y da sentido al tema, particularmente en los planteles del sector privado de educación superior. El trabajo describe los significados que autoridades, profesorado y estudiantado le atribuyen a la acreditación institucional y las principales dificultades enfrentadas, comparando universidades acreditadas y no acreditadas de Santiago de Chile. Los resultados muestran discrepancias entre ambos tipos de universidades y la configuración de orientaciones favorables y refractarias respecto del sistema vigente con variadas consecuencias para su desarrollo y legitimidad social.
\end{abstract}

Palabras clave: acreditación, universidad, privada, profesorado, alumnado, calidad.

\begin{abstract}
The quality assurance by accreditation methods is a challenge facing universities and actors. Empirical evidence in Latin America and Chile is still little, not knowing how to build and provide meaning to the subject, particularly in the private schools of higher education. The research describes the meaning attributed by authorities, teachers and students to institutional accreditation and the main difficulties faced by comparing accredited and non-accredited universities of Santiago de Chile. The results showed discrepancies between the two types of universities and the setting of refractory and favorable guidelines regarding the existing system with various consequences for their development and legitimacy.
\end{abstract}

Key words: accreditation, university, private, teachers, students, quality.

\section{RESUMO}

A garantia de qualidade mediante sistemas de acreditação é um desafio que confronta universidades e atores. A evidência empírica disso na América Latina e no Chile ainda é pequena, desconhecendo-se, por isso, como se constrói e atribui significado ao tema, particularmente nas instituições de ensino superior privadas. Descrevem-se os significados

* El presente artículo forma parte del Proyecto de investigación "Acreditación Institucional y Adaptaciones del Sistema Universitario en la Sociedad del Conocimiento. Una caracterización Cualitativa y Cuantitativa en Universidades Privadas Chilenas", que contó con el auspicio de la Universidad Central de Chile, desarrollado durante los años 2008 al 2010. 
que autoridades, professores e alunos atribuem à acreditação institucional e às principais dificuldades enfrentadas, comparando as universidades não-acreditadas às creditadas de Santiago de Chile. Os resultados mostram discrepâncias entre os dois tipos e a configuração de orientações favoráveis e refratárias a respeito do sistema vigente com várias consequências para seu desenvolvimento e legitimidade.

Palavras chave: acreditação, universitários, particulares, estudantes, qualidade.

\section{INTRODUCCIÓN}

Los sistemas de acreditación institucional, con el objetivo explícito de asegurar la calidad, la transparencia, la utilización eficaz de bienes públicos y privados, así como facilitar la comparabilidad en un mundo cambiante y globalizado, es ya una realidad. Durante los últimos años hemos asistido a una verdadera explosión en cuanto a reglamentación, creación de instituciones y nuevas agencias que regulan los sistemas de educación superior a nivel internacional y nacional. En Chile, como en otros contextos, la inmensa mayoría de las casas de estudio se han sometido al proceso mediante la normativa que establece la CNA. Ello ha implicado que se esté configurando un nuevo campo de estudio multidisciplinar desde las ciencias de la educación, pasando por las ciencias sociales hasta la economía. Actualmente, abundan publicaciones sobre el tema de la acreditación universitaria, sin embargo, se observa que la mayor parte de estos aportes constituyen revisiones bibliográficas con énfasis macrosociales, estudios documentales comparados, institucionales, jurídicos o históricos. Los estudios empíricos que muestren la mirada de los actores que vivencian estas nuevas exigencias y las formas en que las significan son insuficientes para configurar una imagen clara de lo que está actualmente ocurriendo en el heterogéneo mundo universitario. Particularmente, son aún menos frecuentes, aquellas investigaciones que centran la atención en el sector privado y los que toman en cuenta la visión de los principales actores del proceso como el cuerpo directivo, docente y estudiantil.

Asistimos a un giro en los énfasis que marcaron los procesos de certificación y aseguramiento de la calidad en el pasado. Están consolidándose nuevos enfoques que implican profundas modificaciones en los criterios clásicos para evaluar servicios universitarios, tradicionalmente basados en los conceptos de excelencia, eficiencia, eficacia y utilidad. Los nuevos énfasis que han captado la insuficiencia en la mirada puramente normativa, institucional, económica o de gestión vertical, ponen hoy el acento en la pertinencia, la transparencia, las percepciones y significados de los actores, la valorización social y los niveles de involucramiento y participación de los sujetos y grupos (de Miguel, 2003-2004). En ese contexto, nuestro propósito fue conocer los significados que expresan diversos estamentos universitarios sobre el Sistema de Acreditación Institucional, en un conjunto de universidades privadas de la Región Metropolitana de Santiago de Chile, que han obtenido disímiles resultados como dictamen final del proceso.

\section{ESTUDIOS EMPÍRICOS DE ACREDITACIÓN}

En Europa los reportes han girado especialmente en torno a las consecuencias derivadas de la declaración de Bolonia sus logros y contradicciones en los diversos países de la unión. Sobresalen análisis legales sobre los sistemas nacionales, análisis de estadísticas secundarias, especialmente de fuentes estatales o de organismos privados, además de 
reflexiones éticas, filosóficas, pedagógicas y epistemológicas sobre el sustento y el deber ser de los sistemas de acreditación implementado y su devenir futuro. Se constata una cierta desproporción respecto de las abundantes investigaciones aplicados en un plano societal y formal, versus el nivel de análisis empírico de tipo organizacional, referido a las formas en que las universidades han reaccionado y la manera en que de los actores involucrados en el proceso enfrentan estas dinámicas, que en su mayor parte, han sido promovidos por organismos estatales o agencias privadas de alcance nacional e internacional. Esta tendencia se reduce aún más para el caso de las universidades pertenecientes al sector privado, las cuales han constituido un objeto poco indagado, pues el acotado número de investigaciones empíricas existentes ha privilegiado particularmente a las grandes universidades estatales. Los estudios internacionales que levantan información primaria tienden a organizarse sobre dos tendencias fundamentales: aquellos que privilegian el punto de vista organizacional y aquellos otros que toman en cuenta las perspectivas y reacciones de los usuarios, principalmente estudiantes y el estamento docente.

En Estados Unidos existe una larga tradición en cuanto a la aplicación de procedimientos relativos a la acreditación de las instituciones de educación superior, mas, aun así, los especialistas constatan la necesidad de profundizar su importancia. Precisamente, un estudio efectuado en la Universidad de Carolina del Norte verifica que el diseño de las políticas de evaluación de la calidad en universidades resulta más eficiente para la protección del interés público que para garantizar y regular la calidad académica, y que, por tanto, se necesita profundizar la investigación del impacto de estas políticas en el ámbito intra-universitario para perfeccionar las futuras normativas (Hill, 2003). En Suecia, un estudio efectuado por Nilsson y Walhén (2000), mediante un análisis de 27 informes de evaluación institucional y entrevistas a informantes de universidades que se habían sometido al proceso, se concluye que el sistema implementado debe incorporar sustanciales reformas para hacer frente a las deficiencias detectadas. Particularmente se discute la forma de implementación del sistema en una modalidad vertical "desde arriba", que limitó la participación y la voz de actores centrales del proceso situados en las instituciones una perspectiva que no consideró suficientemente la mirada "desde abajo". Adicionalmente, el estudio detecta que al profundizar la profesionalización de la gestión de la calidad a nivel institucional se advierte una tendencia a centrar la atención en los resultados por sobre los procesos, prestando menor atención al grado de involucramiento de los actores, las dinámicas grupales y las diversas transferencias y aprendizajes generados en la interacción.

En un contexto completamente distinto, dado el nivel de desarrollo, es el estudio efectuado por Hopkin (2004), considerando diversos casos de países africanos donde detecta el rol de la agencia externa EQAA, que ha tenido que operar con realidades universitarias muy diversas en el continente. Es así que dependiendo de variables como el tamaño del país, el rol hegemónico de la universidad, el contexto político y las presiones externas, se genera una tipología que da cuenta de disímiles tipos de instituciones respecto de reacciones y adaptaciones ante las exigencias de acreditación. El estudio distingue específicamente tres sistemas: los que denomina "maduros", los "en evolución" y los "embrionarios", concluyendo que entre menos maduro es un sistema de educación superior, es más fácil instalar procesos y metodologías de aseguramiento de la calidad. Strydom y otros (2004) profundizan, para el caso sudafricano, en las relaciones y tensiones derivadas de los cambios que la implementación de estos sistemas trae aparejados 
en la cultura organizacional y las principales fuentes de resistencia en los estamentos administrativos y docentes así como aspectos individuales de tipo refractario que dificultan el establecimiento de una cultura de la calidad. También en la línea organizacional puede nombrarse un reciente reporte de investigación, aunque centrado exclusivamente en el caso de universidades de Nueva Zelandia, donde se indaga una alternativa a los métodos tradicionales, poniendo énfasis en la conformación de comunidades y redes de trabajo, las cuales mediante la instalación de reflexión crítica a nivel de los grupos como metodología sistemática de acción, son capaces de resolver de mejor forma las tensiones que generan sistemas evaluativos centrados exclusivamente en modalidades externas (Jordens y Zepke, 2009).

Otra dimensión de carácter institucional que está comenzando a adquirir relevancia, teniendo en cuenta las consecuencias ante la opinión pública, se relaciona con la difusión digital que cada universidad realiza respecto de los resultados de acreditación obtenidos. Un estudio efectuado sobre una muestra de 150 sitios web de universidades norteamericanas, divididas entre acreditadas y no acreditadas, mostró mediante un análisis cuantitativo que las imágenes utilizadas como publicidad, los mensajes de marketing y los temas de comercialización no están siendo bien aprovechados como elemento de promoción y ventaja comparativa por las universidades acreditadas, a la hora de poner énfasis en el resultado alcanzado, el nivel académico del profesorado y la calidad de la educación que imparten (Adams y Eveland, 2007).

Otros estudios han puesto el acento en el discurso de los líderes y académicos detectando puntos de vista que no siempre se acoplan con las tendencias predominantes promovidas y legitimadas a nivel sistémico. Un estudio que utilizó la metodología de eventos críticos y que comparó el caso del Reino Unido y la República Checa, demostró que existen aspectos de la cultura académica que son normalmente pasados por alto en el aseguramiento de la calidad. Por ello, se requieren indagaciones pormenorizadas dada la diversidad institucional y cultural involucrada, pues se generan tensiones considerables cuando se intenta compatibilizar las exigencias normativas de los sistemas de acreditación con las perspectivas, la motivación y colaboración del cuerpo académico (Mertova y Webster, 2009).

En España, un Foro ANECA, referido a "Los estudiantes y Las políticas de Calidad", se concluye que la participación estudiantil resulta de alta relevancia en los procesos de mejoramiento de la calidad, que ha introducido nuevos temas al debate, que ha permitido ampliar la base de legitimidad de estos procesos al interior de las universidades y que ha instalado la etapa de entrevista con los estudiantes como un aspecto muy importante de los procesos de autoevaluación y acreditación (Aneca, 2005). Otro estudio español sobre docentes permitió describir sus opiniones en el marco de la incorporación de una importante universidad catalana al Espacio Europeo de Educación Superior. Destaca la constatación que un tercio del profesorado se encuentra "poco informado" y "muy dividido", frente a los cambios que dicha incorporación implica, a pesar que la moda de la distribución de las respuestas corresponde a los profesores que valoran positivamente la transformación. Por otra parte, $27 \%$ estima que el cambio significará una mejor formación para el alumno, pero, a la vez, exigirá "mayor dedicación sin reconocimiento" (51\%). El estudio ofrece una tipología con cuatro tipos predominantes frente a la incorporación al EEES: cambio positivo (38\%), cambio negativo (27\%), resistencia negativa $(22 \%)$ y resistencia positiva (14\%) (Masjuan y Troyano, 2009). 
En la perspectiva que enfatiza el punto de vista de los actores, particularmente en universidades privadas, se encuentra el estudio de Iacovidou, Gibbs y Zopiatis (2006) en una universidad privada en Chipre. Si bien se verifica la aceptación del sistema de acreditación y de las estructuras de evaluación de la calidad en las opiniones de los principales interesados, sobre todo en el personal docente y el estudiantado, a la vez se detectaron discrepancias entre ellos, pues mientras los primeros consideraron que los factores cruciales dicen relación con la enseñanza y aprendizaje, los servicios de apoyo y los procesos de aplicación de exámenes, los segundos estimaron que los aspectos más importantes dicen relación con los programas, los cursos, la dinámica y metodología de enseñanza.

La aceptación paulatina, aunque no sin reparos y divisiones, de los sistemas de acreditación y de evaluación de parte de los estamentos universitarios constituye al parecer un fenómeno generalizado. Incluso en zonas distantes o entre grupos étnicos particulares, demuestran este aspecto. Un ejemplo de ello es un reporte acerca del cambio en las percepciones entre 1982 y el 2000, efectuado entre funcionarios de colegios y universidades tribales en Norteamérica, que reconocen transformaciones significativas en la legitimidad del sistema de acreditación aplicado, la importancia atribuida y las funciones que posee (Putman, 2000). Algo similar se aprecia, en cuanto a las modificaciones en las perspectivas de diversos usuarios. Tsui Chung Bing Sum (2002) constata, en Hong Kong, que como producto del impacto de los sistemas de acreditación y su expansión durante la década de los noventa, se fueron generando nuevas percepciones y significados entre los diversos públicos interesados.

En Latinoamérica y Chile el escrutinio se ha focalizado en las concomitantes macrosociales e institucionales y, en la gran mayoría de los casos, las investigaciones se basan en análisis documental, estadísticas secundarias y comparaciones normativas entre sistemas, constituyendo excepciones los estudios realizados mediante levantamiento de datos primarios. Los estudios de actores específicos como docentes y estudiantes no se refieren a los temas de certificación de la calidad, sino que han abordado aspectos más generales como la vinculación y estabilidad laboral de los profesores, los nuevos roles ante el cambio de paradigma de enseñanza-aprendizaje, expectativas laborales, los problemas de la evaluación de los aprendizajes y las diferencias de género, entre otros. Respecto del alumnado, las investigaciones se han centrado en las diferencias socioeconómicas, los procesos de aprendizaje, los perfiles psicosociales, las variables asociadas al rendimiento en carreras universitarias, el acceso a beneficios, la adquisición de competencias profesionales y las trayectorias posteriores y acceso al mundo laboral de los egresados.

Landoni y Romero (2006), en uno de los pocos estudios comparativos sobre educación superior privada a nivel del cono sur de Latinoamérica, afirman que los sistemas de acreditación produjeron impactos importantes en Argentina, Chile y Uruguay, generando isomorfismo y diversificación. Se expresarían tres tipos de isomorfismo; en primer término, el "coercitivo", asociado a las imposiciones legales establecidas desde el Estrado; el "normativo", provocado por la cultura profesional dominante; y el "imitativo", por medio del cual las universidades privadas copian a las instituciones dominantes. Se habrían producido tres impactos fundamentales, relacionados con: a) la restricción de la proliferación de instituciones privadas producto de autorización o licenciamiento de las institucionales de educación superior; b) cierta influencia, aunque limitada, de los procesos de acreditación sobre la homogenización en las instituciones privadas; y c) la 
tendencia de las instituciones privadas para incluir la acreditación como estrategia para obtener legitimidad.

Una línea reciente, pero más específica respecto de la acreditación universitaria, dice relación con algunos reportes que dan cuenta de la experiencia institucional que significó el proceso. Dichos informes constituyen relatos de las principales medidas adoptadas y los planes de mejora implementados, pero no han significado investigaciones sobre datos de primera mano. Ejemplos de este tipo son las publicaciones de Del Castillo (2005), que compara los impactos de la evaluación externa en dos grandes instituciones mexicanas con diverso régimen de funcionamiento. En el caso chileno, se presentan reportes que dan cuenta de las experiencias acreditadoras en universidades específicas como los de Sepúlveda (2004), Mora (2006) y Montes (2004), entre otros.

Entre los estudios de base empírica, se encuentra el relacionado con el proyecto de investigación en políticas de educación superior, donde se sostiene que las actuales 36 universidades privadas del país muestran considerable pluralidad, debido a que en 27 años de existencia se han orientado, desarrollado y diferenciado de manera diversa. Efectivamente, existen distintas misiones fundacionales, orientaciones normativas, segmentación social de sus estudiantes, orientaciones referidas al fomento de la investigación y diferencias en la orientación según principios públicos o privados (Fernández, 2009). Los datos indican que la acreditación se ha transformado en un objetivo muy importante, o "piso mínimo", sobre todo después de la creación del crédito con aval del estado y para alcanzar legitimidad social. Por otra parte, dos de cada tres universidades privadas declaran la investigación como uno de sus objetivos centrales, sin embargo, menos del $30 \%$ participa en los concursos de investigación más importante del país, generándose inconsistencia entre los discursos institucionales y las evidencias.

En un plano más específico, pero que muestra para el caso chileno los ajustes organizacionales que han generado los imperativos derivados de la legislación y la puesta en vigencia de los mecanismos de acreditación en el país, es la investigación efectuada por Rivera, Astudillo y Fernández (2009) a partir de una muestra de universidades que habían desarrollado oficinas de análisis institucional. Se buscaba caracterizar su funcionamiento, operación e impacto en la toma de decisiones de estas unidades. Sobre la base de un catastro, se determinó que de las 61 universidades existentes, 48 habían desarrollado este tipo de instancia sobre todo después de mediados de los años noventa.

Una excepción, en cuanto a tomar en cuenta la voz de los estudiantes, es una exploración sobre los reclamos contra las instituciones de educación superior, donde se caracterizan las principales quejas, los organismos donde se interponen y se derivan algunas implicaciones sobre los posibles conflictos de intereses que se generan (Dooner y otros, 2006). Si bien el contenido de los reclamos no constituye una reproducción de la realidad efectiva en el país, el contenido de las quejas puede entregar pistas acerca de cuáles son los principales temas de preocupación y las dificultades más frecuentes que debe enfrentar el alumnado en su relación con las instituciones a las que pertenecen.

Últimamente se observa una preocupación respecto de un actor que se ha tornado estratégico en el proceso de la acreditación, como es el par evaluador. En Latinoamérica y Chile, asoman dos experiencias, una investigación comparativa en seis países del sistema de aseguramiento de la calidad mediante pares (Marquina, 2006), y otra que aborda el problema de la diversidad institucional de los procesos de acreditación (Sharager y Díaz, 2007). En ambos casos, se ponen de manifiesto las disímiles áreas de tensión que 
debe enfrentar el par evaluador ante realidades organizacionales e institucionales heterogéneas y los problemas, carencias y procesos psicosociales que juegan en los juicios para alcanzar la imparcialidad.

\section{METODOLOGÍA UTILIZADA}

La investigación se estructuró bajo la lógica cualitativa, pues se orientó a la caracterización de los significados sociales (Rodríguez, Gil y García 1999) que poseen los diversos estamentos universitarios respecto de la acreditación institucional. Se utilizó como universo las 21 universidades privadas chilenas del Área Metropolitana de Santiago que tomaron parte en el proceso de acreditación institucional convocado por la Comisión Nacional de Acreditación (CNA) hasta el año 2009. Fueron seleccionadas al azar seis: tres acreditadas y tres no acreditadas. Los nombres de las universidades se han manejado bajo reserva debido a la petición de la gran mayoría de ellas para aceptar formar parte del estudio. Los informantes clave estuvieron vinculados con los estamentos directivo, docente y estudiantil, con el propósito de acceder a sus respectivos significados. Se establecieron criterios relevantes en función de los propósitos del estudio propio de las muestras cualitativas de tipo intencionada (Ruiz, 1996), además de los rasgos típicos recomendados para los informantes clave (Rodríguez, Gil y García, 1999). Los criterios básicos de selección y características consideradas fueron las siguientes: Directivos/as vinculados a los procesos de gestión de cada universidad; Académicos/as que ejercen diversas funciones de Docencia, Investigación y Extensión universitaria; Estudiantes regulares de cursos superiores que participaron del proceso de acreditación o supieron de su aplicación.

En el caso del estudiantado se efectuaron dos grupos focales con el propósito de comparar los resultados que las universidades habían obtenido en sus respectivos procesos de acreditación. Para el primero de los grupos focales se seleccionaron estudiantes de las tres universidades acreditadas y para el segundo se consideraron las tres universidades no acreditadas. Para la obtención del número final de casos, consideramos el criterio de saturación de la información y la necesidad comparativa de los datos (Taylor y Bogdan, 1986; García, Ibáñez y Alvira, 1992).

El análisis de la información se organizó de manera integrada, pues los significados de los tres actores giraron en torno a los mismos temas centrales. Las diferencias surgidas en cada caso se comentan por dimensión, lo que permite una visión comparada así como de los énfasis particulares que en algunos puntos hacían los distintos actores. El análisis privilegia además las diferencias según el resultado obtenido en la acreditación institucional, pues esta variable resultó el aspecto más importante para reconocer las diferencias obtenidas en los relatos de los sujetos. Aspectos como el tipo de carrera, el sexo, la edad, tiempo de docencia o antigüedad de la carrera, no mostraron poseer influencia respecto en los tópicos tratados. 


\section{OBSERVANDO LOS SIGNIFICADOS SOBRE EL SISTEMA DE ACREDITACIÓN INSTITUCIONAL}

\subsection{IDEA GENERAL DEL SISTEMA DE ACREDITACIÓN VIGENTE EN CHILE}

En este tema, los directivos y docentes manifiestan consideraciones definidas y consolidadas, observándose dos grandes visiones, una positiva y otra claramente más crítica del sistema imperante. Si bien la relación no resulta completamente segmentada, se aprecia que los significados positivos tienden a ser sustentados por los directivos y profesores de aquellas universitarias que se encuentran acreditadas y los más negativos por aquellos que no la obtuvieron. La perspectiva favorable indica que si bien el sistema de acreditación chileno es menos exigente que el internacional, contribuye decididamente a establecer parámetros de calidad en la educación superior. Además, es una muestra de que el país está en consonancia con las nuevas realidades emergentes y se está abriendo al mundo y permitiría abrir posibilidades para competir internacionalmente. Sin embargo, también se reconoce que aún tienden a predominar ciertos estándares relacionados con la estructura de universidades tradicionales en el sistema.

“Bueno, yo creo que parte importante del sistema de acreditación es precisamente poder contar con un centro académico moderno, bien administrado, ejecutivo, dinámico, con alternativas para los alumnos no solamente de acceso a la información sino que de prestación de servicios tanto académicos como de otro tipo, creo que es fundamental" (Docente universidad acreditada).

"Las instituciones realmente han internalizado estos procesos, pasaron por una etapa de aprendizaje muy primario con una etapa incluso de reacción en contra o de escepticismo incluso, lo escuche de rectores que decían que eso nunca se va a aplicar aquí o que nuestra cultura no es para eso, en fin, había un escepticismo todo eso se superó" (Directivo universidad acreditada).

"Mira, yo creo, voy a ser positiva con los temas de acreditación, yo creo que lo que permite a las universidades es mirarse y tener el tiempo para auto-reflexionar de sus procesos" (Docente universidad acreditada).

La acreditación constituye, además, según quienes adoptan una posición favorable, un mecanismo que garantiza a los diversos actores estándares generales de tipo educativo y de los procesos universitarios básicos. Se estima que el sistema ha favorecido el desarrollo de ciertas funciones universitarias en el sistema privado como es la investigación y las publicaciones. Sería a la vez un buen parámetro que resulta complementario a los propios procesos internos que realizan las universidades, aunque se reconoce que involucra un arduo trabajo en todos los niveles de la organización.

Nos ha permitido reflexionar, sistematizar poner en valor lo que hacemos, tener claro hacia dónde vamos, involucrar a los profesores" (Directivo universidad acreditada).

"De lo que yo he visto, creo que es un proceso largo, que hay que trabajar mucho, que hay que reunir cualquier cantidad de antecedentes, por lo menos el equipo de trabajo que lidera la acreditación en una facultad, en una universidad, tienen que estar sintonizados todos pal mismo lado, con la misma visión, los mismos objetivos, es mucho, mucho trabajo" (Docente universidad acreditada). 
El punto de vista desfavorable tiende a identificar los procesos de acreditación como un mecanismo aún "en pañales", con instrumentos poco afinados y escasa flexibilidad. Se añade que el sistema se encuentra mal enfocado, pues sería fundamentalmente un negocio que enfatiza lo económico por sobre lo educativo. Sería además un sistema importado, poco adaptado a la realidad local y que fue desarrollado sobre necesidades inmediatas. Se alude, a modo de crítica, los problemas de transparencia que presentaría el proceso y los elevados costos que requiere su aplicación. Al mismo tiempo se afirma que no es posible medir a todas las universidades con el mismo parámetro, y que el sistema estaría estandarizando procesos de realidades universitarias y organizacionales muy diversas. Se añade que habría aún pocas garantías para objetivar la opinión de los pares evaluadores.

"La universidad no fue acreditada, entonces quedamos algo dolidos con el sistema, mi opinión personal es que el sistema debiera funcionar, no como tribunal, sino más bien un proceso de acompañamiento para mejoras" (Directivo universidad no acreditada).

"Yo creo que en Chile es un negociado ya, ahora jmás negociado!, con las instituciones acreditadoras" (Docente universidad no acreditada).

Adicionalmente, es visto como un sistema complicado que otorga poca importancia a los temas de fondo, que aún no cuenta con suficiente información y que presenta un conjunto de problemas de implementación y que puede resultar un arma de "doble filo" en la forma en que se está aplicando, especialmente para las universidades que atienden a los estudiantes de menores ingresos.

"Es un poco engorroso, que la idea es buena, no hay ninguna duda al respecto, es un poco engorroso y me da la impresión de que en algunos aspectos requiere mayor fiscalización, en general" (Docente universidad no acreditada).

"Este tipo de universidades, por ejemplo, son las que educan a estudiantes de sectores que vienen con mas carencias, entonces además les dicen que no están acreditados, es un problema complejo creo yo que tiene que resolverse a nivel de Estado. Es una ley que no está bien hecha, que no ayuda a mejorar la calidad, al final impide que se corrijan esos problemas y se transforma casi en una situación de supervivencia" (Directivo universidad no acreditada).

Todos los estudiantes en cambio tienden a valorizar y considerar positivo el sistema de acreditación institucional vigente en la actualidad, pues da garantías importantes para su propia educación, aunque reconocen manejar poca información y tener bajo interés en el tema. Sin embargo, algunos alumnos estiman que posee elementos comerciales, especialmente los pertenecientes a universidades no acreditadas.

"Hay un nivel alto de ignorancia de la gente que se matricula en las universidades, porque hay mucha gente que no entiende que es lo que significa la acreditación. Es como que les da lo mismo, no saben qué es la CNA" (Estudiante universidad no acreditada).

"Si lo vemos del punto de vista comercial, lo único que busca es como la competitividad, invertir más plata, ¿para qué?, para infraestructura tecnología, para que entren más alumnos, mas plata, y al final el asunto de la acreditación, se transforma muchas veces, en más que un cuento de docencia y esas cosas, en lo que vende" (Estudiante universidad acreditada). 


\subsection{RAZONES POR LAS QUE LA UNIVERSIDAD POSTULÓ A LA ACREDITACIÓN INSTITUCIONAL}

Este aspecto también generó una cierta polarización en las respuestas de los directivos y docentes. No obstante, ambos estamentos reconocen que existe una suerte de obligatoriedad que se impone desde el entorno social como necesidad de adaptarse a los nuevos contextos internacionales e institucionales que generó la normativa de acreditación en el país. Por una parte, se encuentran aquellos que recalcan razones de índole institucional, las cuales fueron parte de decisiones tomadas por las autoridades para entrar en el proceso y de tipo académicas, relacionadas con el establecimiento de mecanismos de aseguramiento de la calidad y excelencia educativa, que permita a los egresados ser competitivos en el mercado y demostrar capacidades para proyectarse en el futuro. Esta perspectiva es compartida fundamentalmente por el estamento docente y directivo, pertenecientes a las universidades acreditadas.

"Son decisiones que se toman a nivel de directorio y se dieron cuenta que era bastante relevante por su nivel de competitividad porque mejora el prestigio y también porque en forma interna la universidad se sentía preparada para un proceso de acreditación" (Directivo universidad acreditada).

"La universidad que no se acredita ahora en Chile no puede funcionar, no puede, prácticamente está muerta ¿te fijas?, o sea eso los alumnos lo saben, o sea no pueden postular a crédito. Da una marca, un sello, en el fondo es que se están haciendo las cosas o sea, no voy a decir bien, pero medianamente bien, aceptable" (Docente universidad acreditada).

En cambio, se detecta una perspectiva opuesta, especialmente prevaleciente entre los no acreditados, respecto de las decisiones para emprender estos procesos en la universidad. En este caso, se afirma que fueron consideraciones más bien prácticas y de conveniencia para sus respectivas instituciones, las que llevaron a involucrarse en el sistema. En este aspecto, los directivos de estas universidades recalcan que las decisiones no fueron las correctas, como tampoco el momento más adecuado.

“...se estimó en su momento que al universidad estaba en condiciones de acreditarse en las tres áreas obligatorias que eran docencia pregrado, gestión institucional y vinculación con el medio y se tiraron a la piscina y te digo, cometieron un error gravísimo" (Directivo universidad no acreditada).

Por su parte, el profesorado de las universidades no acreditadas, si bien reconocen la necesidad de certificar la calidad de los procesos, tienden a ser más críticos con el sistema, esgrimiendo razones relacionadas con meras "estrategias de márketing", o por un cumplimiento formal obligatorio o "mero trámite" que no otorga "ninguna ventaja" en el medio nacional, salvo permitir el acceso a créditos con aval del Estado.

"Porque está obligada, no hay otra razón, está obligada, porque teniendo pedagogías y carreras del área de la salud es obligación" (Docente universidad no acreditada).

"Yo creo que es una manera de mostrarse, de no quedar a un nivel inferior, y hoy en día, lamentablemente, eso es lo que te da el mercado, entonces, el alumno mira: esta universidad está acreditada y ésta no" (Docente universidad no acreditada). 
El alumnado, en tanto, manifiesta no manejar mayores datos al respecto y sólo aquellos que poseen cargos de representación de sus compañeros poseen alguna información adicional.

"Es un nivel de ignorancia, se ve hasta en nuestros pares, yo me enteré porque el año pasado pertenecía al centro de alumnos" (Estudiante universidad no acreditada).

\subsection{PROCESO DE AUTOEVALUACIÓN INSTITUCIONAL E IMPLICACIÓN DEL PROFESORADO, ALUMNADO Y FUNCIONARIOS/AS}

Respecto de este punto también es posible identificar una segmentación entre una visión más positiva, detentada por docentes y directivos principalmente de las universidades acreditadas, respecto de aquellos que no obtuvieron un resultado favorable. En la primera posición se destaca el compromiso e implicación de una parte importante de los miembros de la universidad y el grado de cohesión que generó el proceso, así como el rol que significó la acreditación para identificar de manera clara los puntos débiles y propiciar planes adecuados de corrección. Se destaca la buena conformación de las comisiones y el hecho de que no haya sido un proceso inducido.

"El proceso parte con una decisión de la junta de ingresar al proceso de acreditación. Se crean dos unidades: la comisión de aseguramiento de calidad y la comisión asesora del aseguramiento de la calidad. Para que sea el referente, para que se pueda hablar del tema" (Directivo universidad acreditada).

"En lo administrativo, se fueron creando otro tipo de organizaciones que pudieran mejorar algunas cosas, como mejorar las secretarías de estudio, implementar mejor las direcciones docentes, etc., se han hecho cambios, se notan muchos cambios" (Docente universidad acreditada).

También se relevan algunos elementos menos favorables, tales como la necesidad de mayor información y el hecho que algunos actores como los funcionarios se comprometieron más que el profesorado, muchos de los cuales poseen jornadas parciales o por horas. A la vez se reconoce que los informes de autoevaluación tuvieron un carácter algo general o con resultados dispares en cuanto a calidad y profundidad en las distintas comisiones conformadas al efecto.

"Habian unos informes muy buenos y otros informes muy malos. Y lo que tuvimos que hacer fue tomar toda esa información y volverla a consolidar, prácticamente rehacer todos los informes y consolidar un informe coherente" (Directivo universidad acreditada).

"Una universidad que descansa fundamentalmente en una plataforma docente de "profesores taxi", que tienen compromisos puntuales a partir de ciertas cátedras, pero que no tienen los tiempos necesarios y suficientes como para poder formar parte de una comunidad universitaria en términos mucho más complejos de formar parte de este análisis, creo que es uno de los problemas" (Docente universidad acreditada).

La visión que pone énfasis fundamentalmente en los aspectos negativos, destaca que el proceso estuvo muy centralizado y contó con baja o escasa socialización, dispuso de muy poca información y no se estructuró mediante los canales adecuados. Adicionalmente, se afirma que se generaron informes autocomplacientes, que no detectaron adecuadamente 
las verdaderas fortalezas y debilidades, lo que influyó para que no se tomaran las medidas requeridas y urgentes en ese momento.

"Internamente tuvo mucho impacto, se llevó a cabo una gestión que yo no la comparto, porque una vez que se conoce la resolución comienza un crecimiento inorgánico, comienza a crearse sedes, varias sedes en el norte y en el sur y carreras en el área social, área técnica, área economía y de alguna manera el proyecto se empieza a abrir pero también a perderse el proyecto original y no se asumen las grandes críticas que se hacen en el dictamen de la CNA" (Directivo universidad no acreditada).

"Hubo problemas, tanto con los pares evaluadores, como después con la comisión, tuvimos problemas con la autoevaluación, porque nosotros hay como mucha cuestión de piel, entonces, la evaluación a lo mejor la vieron que era muy buena por el lado de nosotros, era muy complaciente, y por eso mismo no nos permitió ver defectos" (Docente universidad no acreditada).

Se reconoce, no obstante, que la experiencia permitió activar una serie de procesos que no estaban implementados y sirvió como una toma de conciencia y para el mejoramiento de algunas deficiencias principalmente en la infraestructura disponible, aunque significó una recarga laboral importante para los participantes.

"Los aspectos positivos es que la gente empezó a comunicarse más, a abrirse más, a poder explicitar mas su perspectiva y visión crítica, a establecer relaciones más francas y claras, la comunidad se articuló completamente, los alumnos con los docentes, los docentes con los directivos, los directivos con las autoridades de la universidad, etcétera. Y el aspecto entre comillas negativo es el exceso de trabajo" (Directivo universidad no acreditada)

En este punto, los estudiantes nuevamente manifestaron nula información, pues señalan no tener recuerdo del proceso. Algunos agregan que las autoridades tampoco informaron adecuadamente.

\subsection{CONSIDERACIÓN SOBRE LA VISITA DE PARES EVALUADORES}

Los relatos de directivos y docentes reflejan en este tema situaciones dispares que no se agrupan necesariamente en relación a la dirección de los significados ni al tipo de universidad o resultado de acreditación obtenido, pues refleja fundamentalmente la experiencia individual vivida en cada caso. Es así que tampoco se presentan relaciones o consensos fuertes, aunque los profesores manejan claramente menor información al respecto o definitivamente no la disponen. Los estudiantes de universidades acreditadas como no acreditadas declararon en su totalidad no conocer ni recordar este hecho.

"Fue para mi gusto rápida, estuvieron una semana acá en la escuela yo los debo haber visto dos veces. Sí, nada más que dos veces. No, simplemente vine más peinado no más que otros días (risas). O sea lo digo como broma pero es un día igual que todos" (Docente universidad acreditada).

Un aspecto que parece organizar las narraciones es la tendencia a revelar una preparación del proceso de visita de pares, frente a otra posición que señala, de manera explícita, que no se hizo ninguna acción especial en ese sentido. En el primer caso, se indica que las autoridades junto con informar de manera amplia al cuerpo académico y administrativo, procedieron a efectuar una simulación con la ayuda de un experto, lo que habría implicado un esfuerzo especial respecto de una situación normal. Otros informantes 
revelan que en sus universidades no habría existido un proceso de preparación, porque hubo participación constante.

"Una vez entregado el informe de autoevaluación, la idea era hacer una comunicación o socialización. En el aula magna hicimos una presentación explicando qué habíamos hecho, toda la universidad se socializó con el informe, estaban presentes desde la junta directiva, hasta los cargos más bajitos. Hicimos una simulación, y en esa simulación, conseguimos la participación de otro par evaluador externo" (Directivo universidad acreditada).

"No, o sea, nos dijeron la fecha justo que iba[n] a ir, ordenaron los grupos que teníamos que ir, pero que dijeran lo que teníamos que hablar, o mucho menos, nada, yo por lo menos nada" (Docente universidad no acreditada).

En dos universidades no acreditadas, se advierte una carencia de información sobre los detalles de la visita anterior, resultado que habría sido recibido con sorpresa por la comunidad.

"No estuve ahí, yo solamente fui a una reunión final en la que, finalmente, y el proceso estaba en un avance muy importante, pero no estuve en ese proceso, no lo conocí" (Docente universidad no acreditada).

"Fue súper sorprendente, porque alguna manera manifestaron que la universidad estaba bien, y la sorpresa fue cuando llegó el informe final y dijeron que la universidad no estaba acreditada" (Docente universidad no acreditada).

En el relato de algunos entrevistados se refleja que habría existido una indudable expectación y tensión junto a la sensación de sentirse rindiendo una especie de examen durante estas visitas.

"Fue como nervioso, esa visita de los pares, si nosotros tenemos dos sedes Santiago y Concepción, estuvimos todos informados cómo y cuándo vendrían a la universidad. Fíjate tengo la impresión que es muy serio todo, o sea, hubo una preparación, no como un panfleto que tú tenías que repetir, sino quién[es] eran estos pares, a qué venían, con quiénes se entrevistarán" (Directivo universidad acreditada).

"La visita fue una buena en el sentido que se focalizó en los aspectos que debía focalizarse, o sea, en las áreas de trabajo, se aprendió del proceso, al someterse a este examen por decir así externo, es sano, es bueno para la institución" (Directivo universidad acreditada).

"El comité de pares asume el rol más bien de un inspector de impuestos internos que viene a ver dónde está lo malo y dónde puedo hacer caer el machete sobre la institución y a castigarla, es decir, en el fondo te pillé" (Directivo universidad acreditada).

Entre los directivos las opiniones tienden a ser más consistentes al considerar que la visita de los pares fue correcta, positiva, cordial y se enfocó en los aspectos que correspondía, a pesar de la tensión reinante en la ocasión.

"Hicimos un esfuerzo en eso y nos pareció que la visita transcurrió con absoluta normalidad no hubo absolutamente ningún problema que ellos nos señalaran, hicimos todo lo que ellos nos pidieron, los llevamos a visitar todas las instalaciones de la universidad, se reunieron con todas las autoridades con quienes ellos quisieron" (Directivo universidad no acreditada). 
Entre el profesorado las opiniones son en general positivas, pero con un menor grado de información, dado que no todos ellos participaron de manera igualmente activa en el día de la visita o no se informaron especialmente al respecto. Sólo uno de los casos entrevistados señala que la visita de los pares fue poco rigurosa si se compara con la de tipo internacional en que participa su universidad en particular.

\subsection{CONSIDERACIÓN SOBRE EL RESULTADO OBTENIDO EN EL PROCESO}

Las respuestas en este tema presentan cierta diversidad por universidad y actor, dividiéndose entre quienes no quedaron completamente conformes con el resultado, aquellos que lo consideraron adecuado y ajustado a la realidad de la universidad y, finalmente, aquellos que lo evaluaron como muy bueno. Estas apreciaciones son compartidas tanto por directivos como por docentes. Entre los primeros se encuentran las consideraciones en dos universidades acreditadas de mayor antigüedad que esperaban más años de acreditación y que enfrentaban proceso por segunda vez, pero que de todas formas estiman que lo logrado constituye un mérito. Incluso en el caso de una de ellas, se señala que se produjo un proceso de apelación por considerarse que la CNA no había ponderado todos los aspectos señalados en la autoevaluación institucional.

"Esto es muy personal, yo no quedé de acuerdo con la cantidad de años que nos dieron, efectivamente la universidad tiene una cantidad importante de falencias, y si no se pudieron resolver en el plazo en donde trabajamos en el proceso, nosotros las colocamos todas en el plan de mejora, entonces siempre fueron detectadas, fuimos muy críticos" (Directivo universidad acreditada).

En el segundo grupo se encuentran los directivos y docentes entrevistados de las tres universidades no acreditadas seleccionadas, quienes reconocen la existencia de falencias importantes. Incluso opinan que la institución no debió haberse presentado al proceso, porque no existían las condiciones necesarias, que los informes de CNA fueron negativos, pero veraces, transformándose en un insumo relevante para enfrentar las falencias detectadas.

"Me parece un fundamento veraz, también generoso, en el sentido que creo que fue un muy buen informe porque nos ha servido para mirarnos más profundamente" (Docente universidad no acreditada).

"O sea, yo creo que la comisión debió haber tenido todos sus criterios bien ajustados para tomar esta determinación, yo creo que es un desafío justamente, hay que dar una respuesta positiva, tenemos que mejorar en muchos aspectos" (Docente universidad no acreditada).

Los estudiantes de todas las universidades, manifestaron una opinión más uniforme, fundamentalmente en acuerdo con el resultado obtenido, pues las falencias detectadas eran según ellos evidentes, aunque en este tema, también se observa desconocimiento por los detalles del dictamen oficial.

"No tengo idea, cuantos años le dieron a la universidad" (Estudiante universidad acreditada).

"Es que tampoco andamos hablando así del tema, no es tema para nosotros" (Estudiante universidad acreditada). 
"Por lo menos yo creo que mi universidad estuvo bien no haberla acreditado" (Estudiante universidad no acreditada).

"En mi universidad estaban acreditando al mismo tiempo con una universidad extranjera, entonces ésa era como más importante, entonces ésa fue como la que se llevó todo, es más, uno se metía a la página y salía gigante: jacreditados en tal país! y jahora hay intercambios!, entonces la internacional era la importante" (Estudiante universidad acreditada).

En relación con el informe emitido por los directivos, claramente manejan mayores detalles, pues dicho documento ha sido objeto de discusión y análisis al interior de diversas unidades. En cambio, los docentes y estudiantes, en su gran mayoría, indican que no lo conocen, la difusión no ha sido la adecuada o no tienen un interés especial en ello.

"La universidad mandó esos mail como del resumen de la semana, pero yo nunca abro esos mail" (Estudiante universidad acreditada).

\subsection{IMPACTO DEL RESULTADO OBTENIDO}

Los relatos sobre el impacto provocado por el resultado se dividieron nuevamente entre las universidades acreditadas y las no acreditadas. Entre las primeras coinciden en que las principales consecuencias fueron en general positivas, aunque algunas no se han manifestado completamente. Habría servido para elevar el número de matrículas, los puntajes de los que ingresan y un mejor posicionamiento en el mercado, eliminando, además, prejuicios respecto de las universidades privadas.

"Después de estos procesos hemos ganado prestigio, hemos tenido una admisión muy buena, ha ido creciendo la admisión y el puntaje PSU, se ha ido sofisticando un poco también el alumno que ingresa y eso ha sido una ganancia" (Directivo universidad acreditada).

"El impacto interno es que hoy día tenemos catorce mil estudiantes como universidad, en nuestra carrera este año ingresaron ciento noventa alumnos, ahí está la importancia de la acreditación de lograr mejores resultados y mantenerse acreditados" (Docente universidad acreditada).

Además, se agrega como efecto positivo el mejoramiento del clima y la cohesión, el incremento en las oportunidades y vínculos con el entorno, la focalización del plan de mejora y el incremento general de la calidad en los diversos procesos. Los jóvenes de las universidades acreditadas, recuerdan que las autoridades informaron poniendo cierto énfasis en el resultado como un logro para la institución, aunque efectúan menos relaciones de significado que los profesores y directivos.

"El rector se puso a hablar en el patio central y les regalaron no se que a cada alumno, no si fue show, se notó. Si también cambiaron el logo de la universidad" (Estudiante universidad acreditada).

Entre los no acreditados, predominan las opiniones que enfatizan consecuencias negativas para la universidad, pues habría impactado en una disminución de los matriculados y generado la perplejidad en los diversos estamentos. Por otra parte, se reconoce que el resultado adverso provoca la imposibilidad de postular a créditos con aval del Estado. 
"Fue un impacto brutal en términos *de tanto de matrícula, por ejemplo, como de recepción, de la imagen, los profesores, los estudiantes, yo creo que la universidad entra en una suerte de shock los primeros meses" (Directivo universidad no acreditada).

"Una no acreditación, realmente puede afectar y llevar al límite a una institución" (Directivo universidad no acreditada).

"Los alumnos anduvieron como un mes así como...parecían fantasmas, o sea, exijo una explicación, ¿qué pasó?” (Docente universidad no acreditada).

Los estudiantes recuerdan que, conocida la no acreditación de sus respectivas universidades, se generaron una serie de movilizaciones que buscaban presionar para que la universidad realizara los cambios necesarios para alcanzar un mejor resultado en el futuro.

"Lo que pasó en nuestra universidad, medicina se fue a paro y solucionados los problemas y ahora se fue a paro enfermería" (Estudiante universidad no acreditada).

"Nosotros hicimos toma, porque en las condiciones que estábamos eran deplorables, eso fue después del resultado. $Y$ en ese punto, aparte de la acreditación *fueron como lo que gatilló que se diera la situación [en] que estábamos" (Estudiante universidad no acreditada).

Se reconoce, no obstante, sobre todo por parte del grupo de docentes y directivos, que el resultado adverso también permite generar conciencia para agilizar y reordenar procesos y la generación de un espíritu de superación.

"Se están tomando varias medidas correctivas, el último añ,o por ejemplo, a nivel de pedagogía, estamos cerrando alrededor de 19 carreras, qué te quiero decir con esto, que en el último año la idea era racionalizar la oferta y entregar una oferta de calidad sustentable académica y financieramente" (Directivo universidad no acreditada).

"Todos, el cuerpo de coordinadores, docentes y alumnos trabajaron unidos por los mismos fines, hubo una cohesión, y externo, que no nos acreditaron y hubo presión y preocupación de los alumnos por saber cuáles fueron las causas y si su título iba a valer" (Docente universidad no acreditada).

\subsection{CAMBIOS QUE DEBEN EFECTUARSE AL SISTEMA DE ACREDITACIÓN INSTITUCIONAL}

Este tema presenta consistencia entre los relatos de directivos y docentes así como entre las diversas universidades, tanto acreditadas como no acreditadas, salvo pequeñas diferencias puntuales. Los cambios que se sugieren, pueden agruparse en aspectos relativos al sistema en general, la transparencia de los procesos, los pares evaluadores y ciertas consideraciones relacionadas con el tipo de universidad. Se señala que debería existir un reconocimiento y validación del sistema en el concierto internacional, mejorar la claridad y pluralidad de los criterios de evaluación, disminuir la burocratización y generar mayor comprensión para los proyectos educativos particulares. Se añade que debería haber mayor preocupación por las agencias acreditadoras, lo cual revela imprecisión en el manejo de la institucionalidad vigente, pues, en el caso chileno, estos organismos abordan la acreditación de las carreras. 
"Está haciendo falta que la CNA tenga de alguna manera algún tipo de reconocimiento internacional, o sea tú te acreditas hoy día ante la CNA y pasas a ser una universidad acreditada y tienen todos los logos, pero tu sales de aquí del país y fuera del país no te conoce nadie" (Directivo universidad acreditada).

"Sería interesante que las acreditaciones tengan estándares mínimos, pero también ser flexibles a los propios proyectos educativos de las universidades. Pretender que todos seamos Universidad Católica, Universidad de Chile, tampoco nos parece, sino respetar la identidad de las universidades" (Directivo universidad acreditada).

Por otra parte, se sugiere introducir mayor transparencia en la selección de los pares evaluadores, aumentar su número y una mayor énfasis por mejorar los procesos de entrenamiento de estos expertos, pues habría diferencias importantes en los niveles de inducción y experiencia necesaria.

"Hay una falencia de evaluadores y un mayor entrenamiento de esos evaluadores, esto no es menor porque al haber pocos tienen que estar recurriendo a los mismos, si tú haces una estadística en este momento y esto pasa a una segunda falencia o cosa por hacer o superar es que en esta ampliación debe integrarse más en los distintos sectores de las instituciones universitarias" (Directivo universidad acreditada).

Cabe indicar que el cuerpo académico tiende a ser más crítico en algunos de los aspectos antes indicados por lo directivos.

"Veo que es un nuevo negocio volver a lo que antes tenían los colegios profesionales" (Docente universidad acreditada).

"Ojo quién va, a qué universidad, ojo con las agencias acreditadoras, que son agencias que se están configurando para ganar plata. Entonces tú te haces amigo de la agencia acreditadora, entonces es un chiste" (Docente universidad acreditada).

"A mí me gustaría que hubiera más presencia de pares de las universidades privadas que tuvieran una mirada más complementaria. Esperaría que los niveles de exigencia también fueran altos, y que no fuese un trámite de que la acreditación no se transformara en un instrumento burocrático, de llenar formularios. Yo haría que los pares evaluadores vinieran, idealmente, en distintos momentos, o que permanecieran más días" (Docente universidad no acreditada).

Los estudiantes, si bien estiman que es positivo que exista el sistema de acreditación, tienden a diferenciarse entre los acreditados y no acreditados. Los primeros ponen el acento en aspectos relacionados con las exigencias educativas a las universidades, en cambio, los segundos subrayan el carácter economicista del sistema.

"Debería haber más exigencia para los profesores, para acreditar a los profesores, que tengan un grado mayor académico, el resultado obtenido por los alumnos, tiene que ser vinculante con la evaluación docente" (Estudiante universidad acreditada).

"El tema de la acreditación va totalmente arraigado al tema de lo comercial" (Estudiante universidad no acreditada). 


\section{CONCLUSIONES}

El sistema de acreditación institucional vigente en el país es observado mayoritariamente como una necesidad, con seriedad en los procesos, que genera efectos en general positivos, que refuerzan la colaboración interna y las capacidades de diagnóstico, mediante la configuración de equipos de trabajo que promueven la autorregulación de las universidades del sector privado. Ello no significa un respaldo unánime o un apoyo irrestricto al modelo, por el contrario, se aprecian disparidades importantes en muchas de las dimensiones analizadas. Estos significados se resumen en el siguiente esquema:
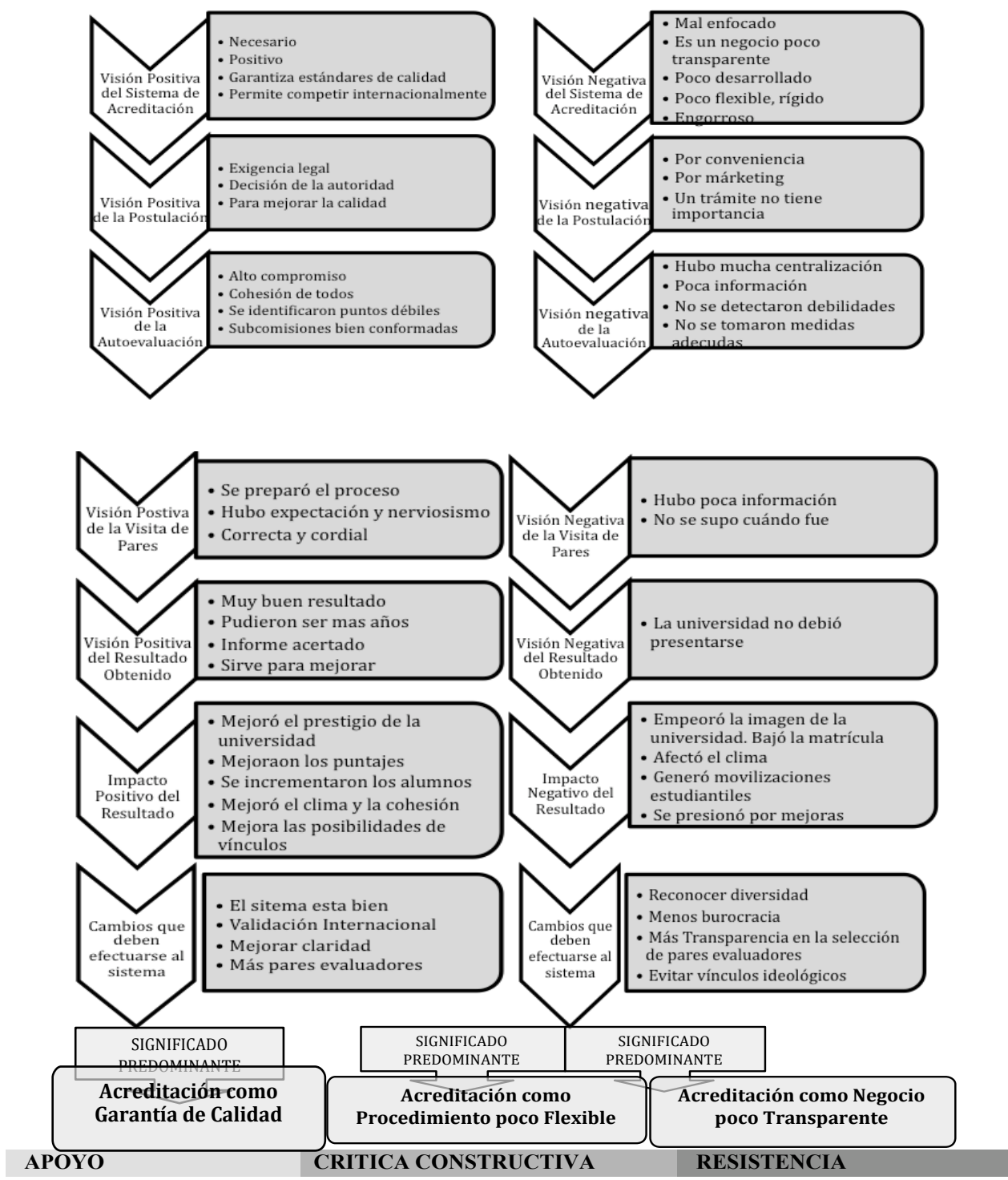

Tabla 1. Significados sobre el sistema de acreditación 
Se configuran dos polos de significados principales, uno positivo y otro negativo y tres perspectivas bien diferenciadas. A estas miradas las denominamos: Acreditación como Garantía de Calidad, Acreditación como Procedimiento poco Flexible y Acreditación como Negocio poco Transparente. La primera se encuentra sustentada casi en su totalidad por el cuerpo directivo, docente y estudiantado de las universidades que fueron acreditadas. En cambio, las concepciones negativas son sostenidas especialmente por quienes pertenecen a las universidades que no obtuvieron la acreditación.

Efectivamente, en la mayoría de los temas que se abordaron, se detectan diferencias contrastantes. Quienes la conciben como Garantía de Calidad, le otorgan al sistema un claro apoyo, asumiéndolo como necesario y bien enfocado o que requeriría sólo ajustes menores. Los significados negativos dan lugar a dos visiones, una de ellas que denominamos crítica constructiva, que sin rechazar el sistema, estima que es preciso incorporar cambios importantes. También se configura una postura refractaria, dando cuenta de una orientación de resistencia, que recalca las distorsiones económicas e ideológicas que esta visión atribuye al modelo.

Los resultados mostraron, adicionalmente, una diferencia apreciable en el grado de conocimiento que los estamentos poseen respecto de la acreditación institucional. Este aspecto llama la atención, pues si bien se espera que los intereses del alumnado se encuentren principalmente orientados hacia su propia carrera, sorprende de todas formas el fuerte desconocimiento de aspectos clave del proceso. Ello constituye una discrepancia respecto del modelo que se busca aplicar en Chile, que tal como lo plantea la Comisión Nacional de Acreditación CNA, intenta constituirse en un mecanismo de involucramiento de los diversos actores, con altos niveles de información y transparencia como garantía para la opinión pública, los estudiantes y sus familias (CNA, 2007). Estos resultados son consistentes con lo planteado por diversos especialistas que estiman que en Latinoamérica y en Chile, no se ha logrado afianzar la acreditación ni se ha configurado aún una verdadera cultura de la calidad (Brunner, 2008; Pires y Lemaitre, 2008). En el decir de Hopkin (2004), correspondería a un sistema "en evolución".

Entendemos que si bien el resultado alcanzado constituye una poderosa razón para dar sentido y organizar los significados que los diversos actores adjudican al sistema, no es el único elemento que influye en la estructuración de las perspectivas. Los factores que dicen relación con la orientación previa de la propia universidad, los cuales probablemente atraen a un profesorado y alumnado más acorde con determinadas posturas doctrinarias y las condiciones materiales, en cuanto a tamaño, disponibilidad de infraestructura y equipamiento, son elementos que catalizan una toma de posición determinada. A nuestro entender, ello predispone a determinadas concepciones favorables o críticas, con anterioridad a que la institución tome parte en un sistema de aseguramiento de la calidad. La presencia de distintas perspectivas, según el tipo de resultado obtenido, advierten sobre aspectos preocupantes que deben ser abordados a la brevedad por las diversas instancias involucradas. Independiente de las modificaciones legales o reglamentarias que puedan introducirse a nivel institucional para mejorar la calidad, ellas serán insuficientes si no recogen la variabilidad que muestra el actual sistema universitario chileno y la forma en que los diversos grupos le asignan sentido en su cotidianeidad.

Los juicios más críticos junto a la desinformación y confusiones respecto del modelo de acreditación vigente, si bien son minoritarios, pueden dar lugar en el futuro a dificultades para obtener un mayor compromiso de los actores o eventualmente, poner en entredicho 
la legitimidad de los procedimientos que se aplican. Ello nos habla de un sistema que no se ha consolidado, que busca introducir nuevas normas, lenguajes mecanismos y en medio de dinámicas ya arraigadas, de concepciones diferenciadas y de resistencias que dan cuenta de concepciones universitarias variables y juegos contrapuestos de intereses. Aunque todos los grupos reconocen la vigencia y la seriedad de los informes que emite la CNA, existen amenazas que podrían afectar la estabilidad del sistema. El hecho que entre los que no han sido acreditados predominen críticas constructivas que demandan más agilidad y flexibilidad al modelo, pero también rechazo ante el mismo, por estimar que el sistema adolece de sesgos económicos, constituye un riesgo que los organismos pertinentes deberían considerar en la perspectiva de afianzar el modelo acreditador e incrementar sus niveles de validación y legitimización social. Aspectos que dicen relación con los niveles de burocracia, la falta de flexibilidad para considerar distintos tipos de universidad y la transparencia, especialmente en lo que respecta a los pares evaluadores, podrían afectar a su vez la aceptación de los procedimientos como dispositivos adecuados para medir, con un parámetro único, a todas las universidades del país.

Entre las universidades privadas estudiadas no se constituye una cultura de la calidad en toda su expresión, en la medida que las demandas por mejoras al sistema acreditador tienden a no auto-incluír a las propias instituciones de educación superior. Desde la perspectiva de los actores, el perfeccionamiento del sistema no parece ser un problema que deba afrontar la universidad como organización, sino que estaría en el entorno y, por lo tanto, sería responsabilidad del Estado, la CNA o el Ministerio de Educación tomar las medidas correctivas. Se observa así una cierta disociación, ya que existiendo un reconocimiento, prevalece a la vez un bajo nivel de compromiso con el sistema. El hecho que autoridades universitarias, docentes y estudiantes no sientan como propio el modelo acreditador, puede tornar menos eficaces las iniciativas destinadas a su perfeccionamiento futuro.

Los hallazgos implican también retos de importancia respecto de los procesos internos en cada universidad. Si bien la gran mayoría asume la necesidad de que la organización lleve a cabo estos procesos de manera exitosa, subsisten carencias de diversa índole. Los alumnos de todas las universidades saben muy poco de la acreditación, sus distintas fases y las consecuencias a las que da lugar. En algunos casos de universidades que no fueron acreditadas se generaron movilizaciones estudiantiles que exigían mejoras, después de conocidos los resultados. Entre las dificultades más importantes identificadas en estos casos, se encuentran la centralización excesiva por parte de las autoridades superiores, las falencias de información en diversas etapas, la conformación inadecuada de las subcomisiones, la escasa implicación del profesorado y, particularmente, del alumnado en la autoevaluación y la incapacidad para identificar debilidades y corregirlas a tiempo. Las críticas a la flexibilidad del sistema de acreditación están, por el momento, relacionadas con los estándares homogéneos que según algunos se aplicaban a sus universidades. Estos criterios, desde la perspectiva de los actores más refractarios, dejarían poco espacio para el desarrollo de proyectos educativos distintos pues, adicionalmente, los pares evaluadores emitirían juicios basados aún en el modelo de universidad tradicional chilena, imponiendo, así, determinados rasgos propios del sistema público al sistema privado.

El riesgo de que la acreditación pueda significar una posible homogenización en el sistema, con una estructuración monótona de las universidades frente a las demandas del entorno, sigue vigente. Ello puede derivar en una disminución de las capacidades adaptativas 
del sistema universitario para enfrentar las transformaciones y permanentes exigencias provenientes de la globalización y la sociedad de la información y el conocimiento. Enfrentar estos riesgos requerirá de reflexión teórica e investigación empírica, ofreciendo una visión más integral, capaz de generar propuestas para dirigir inteligentemente el cambio, donde el cultivo del saber posea un rol protagónico para flexibilizar procedimientos, incorporar mecanismos de consultas, reclamación eficiente y con facilidad de acceso, procurando perfeccionar la acreditación y afianzar sus niveles de legitimación social.

\section{REFERENCIAS BIBLIOGRÁFICAS}

Adams, J. y Eveland, V. (2007): Marketing online degree programs: How do tradicional-residential programs complete? Journal of Marketing for higher education, Vol. 17, $\mathrm{N}^{\circ} 1$.

ANECA (2005): Los Estudiantes y las Políticas de Calidad. IV Foro ANECA. 20 de Octubre, Madrid: ANECA.

Brunner, J.J. (2008): El Proceso de Bolonia en el horizonte latinoamericano: límites y posibilidades. Revista de Educación, Número Especial.

De Miguel, M. (2003-2004): Evaluación Institucional versus Acreditación en la Enseñanza Superior”. Contextos Educativos, 6-7, 2003-2004. Edición electrónica, Dialnet.

Del Castillo, G. (2005): Dos modelos diferenciados de configuración institucional bajo el impacto de la evaluación externa: la UAM-A y la UIA. México: Ed. ANUIES.

Dooner, C., Guevara, M., Lagos, F. y Mena, P. (2006): La voz de los usuarios. Reclamos contra las instituciones de educación superior. Calidad en la Educación, $\mathrm{N}^{\circ} 25$.

Fernández, E. (2009): Perspectivas de futuro de las universidades privadas fundadas con posterioridad a 1980. En J.J. Brunner y C. Peña (eds). Reforma de la Educación Superior. Santiago: Ediciones Universidad Diego Portales.

García, M., Ibáñez, J. y Alvira, F. (1992). Análisis de la realidad social. Métodos y técnicas de investigación. Madrid: Ed. Alianza.

Hopkin, A. (2004): Frame factor and quality assurance agency in an embryonic higher education system". Quality in higher education, Vol.10, N³.

Hill, D. (2003): An Institucional Perspectiva on higher education policy: The case of academia quality assurance". En J. Smart (Ed.), Higher Education: Handbook of theory and Research, Vol. XVII.

Iacovidou, M., Gibbs, P. y Zopiatis, A. (2009): An exploratory use of the stakeholder approach to defining and measuring quality: The case a Cypriot Higher Education Institution", Quality in Higher Education, Vol. 15, $\mathrm{N}^{\circ} 2$.

Jordens, Z. y Zepke, N. (2009): A network approach to curriculum quality assessment. Quality in higher education, Vol.15, №3.

Landoni, P. y Romero, C. (2006): Aseguramiento de la calidad y desarrollo de la educación superior privada: Comparaciones entre las experiencias de Argentina, Chile y Uruguay". Revista Calidad de la Educación, n. 25.

Nilsson, K. y Wahlén, S. (2000): Institutional response to the Swedish model of quality assurance. Quality in Higher Educa $\Psi \mathrm{P}_{n}$, Vol. 6, $\mathrm{N}^{\circ} 1$.

Marquina, M. (S/F): La evaluación por pares en el escenario actual de aseguramiento de la calidad de la educación superior: Un estudio comparativo de seis casos nacionales. Textos electrónicos CONEAU. www.coneau.edu.ar/archivos/1331.pdf

Masjuan, J. y Troyano, H. (2009): Incorporación de España al Espacio Europeo de Educación Superior: El caso de una Universidad catalana". Revista Calidad de la Educación, n.31. 
Mertova, P. y Webster, L. (2009): The academia voice in English and Czech higher education quality. Quality AssuraOce in education. Internalibnal Perspective, Vol. 17, N².

Montes, F. (2004): Experiencia de Licenciamiento de la Universidad Alberto Hurtado con el Consejo Superior de Educación. Revista Calidad en la Educación, $\mathrm{N}^{\circ} 21$.

Mora, A. (2006): Impacto de los procesos de acreditación en la Universidad de Concepción. Revista Calidad en la Educación, n. 24.

Pires, S. y Lemaitre, M.J. (2008). Sistemas de acreditación y evaluación de la educación superior en América Latina y El Caribe, en CRES Tendencias de Educación Superior en América Latina y el Caribe. Caracas, Conferencia Regional de Educación Superior - CRES, IESALC- UNESCO.

Putman, T. E. (2000): Tribal College an University Accreditation: A Comparative Study. Dissertation. Abstracts International, $\mathrm{N}^{\circ} 62$.

Rodríguez, G., Gil, J. y García, E. (1999). Metodología de la Investigación Cualitativa. Málaga: Ed. Aljibe.

Ruiz, P. (1998): La Universidad y los Poderes Públicos. En Porta, J. y Lladonosa M. (coords.), La Universidad en el Cambio de Siglo. Madrid: Ed. Alianza.

Scharager, J. y Díaz, R. (2007). Aceptación de la diversidad institucional en los procesos de acreditación: La posición del par evaluador. Calidad en la Educación, $\mathrm{N}^{\circ} 26$.

Sepúlveda, C. (2004): Primera Experiencia de Acreditación Institucional en la Universidad de Chile. Calidad en la Educación, $\mathrm{N}^{\circ} 21$.

Strydom, J., Z, N. y Murria, L. (2004): Quality, culture and change. Quality in higher Educa UPn, Vol.10, $\mathrm{N}^{\circ} 3$.

Taylor S. y Bogdan R. (1986). Introducción a los Métodos cualitativos de Investigación Social. Madrid: Ed. Paidós.

Tsui Chung Bing Sum, C. (2002). Quality in higher education: Policies and practices. A Hong Kong perspectivF. Intruduclibn and Research Approach. The University of London, United Kingdom. 